\title{
Recurrent glomerulonephritis following renal transplantation and impact on graft survival
}

S. H. Jiang ${ }^{1,2 \dagger}$, A. L. Kennard ${ }^{1 \dagger}$ and G. D. Walters ${ }^{1,2,3,4^{*}}$

\begin{abstract}
Background: Recurrence of primary glomerulonephritis in the post-transplant period has been described in the literature but the risk remains poorly quantified and its impact on allograft outcomes and implications for subsequent transplants remain under-examined. Here we describe the rates and timing of post-transplant glomerulonephritis recurrence for IgA nephropathy, focal segmental glomerulosclerosis, mesangiocapillary GN and membranous GN based on 28 years of ANZDATA registry transplant data.

Methods: We investigated the rates of GN recurrence and subsequent graft outcomes in 7236 patient from 28 years of ANZDATA transplant registry data. Data were analysed in R, using Kaplan Meier Survival analysis and adjusted analyses performed using Cox Proportional Hazards methods. A competing risk model was also analysed.

Results: GN recurrence occurred in $10.5 \%$ of transplants and was most common in mesangiocapillary GN. Median time to recurrence was shorter for FSGS compared to IGAN. GN recurrence was less common in patients over 50 years of age and after unrelated kidney donation. We identified a significantly higher risk of recurrence in secondary grafts following recurrence in a primary allograft for FSGS (RR 5.70, 95 Cl: 2.41-13.5, $p<0.001$ ) but not IGAN, MCGN or MN. At 10 years, recurrence occurs in 8.7, 10.8, 13.1, and 13.4\% of allografts for FSGS, IGAN, MCGN and MN respectively. In all GN, recurrence significantly reduced death censored graft survival at 5 and 10 years.
\end{abstract}

Conclusions: GN recurrence occurs in a minority of patients at a significantly different rate for each GN. After a recurrence, there is no evidence for an increased risk of further recurrence in a subsequent graft except in FSGS.

Keywords: Glomerulonephritis, Transplant, Disease recurrence

\footnotetext{
* Correspondence: giles.walters@act.gov.au

${ }^{\dagger}$ S. H. Jiang and A. L. Kennard contributed equally to this work.

${ }^{1}$ Department of Renal Medicine, The Canberra Hospital, PO Box 11, Woden,

Canberra ACT 2605, Australia

${ }^{2}$ Department of Immunology and Infectious Diseases, John Curtin School of

Medical Research, Australian National University, Canberra, Australia

Full list of author information is available at the end of the article
}

(c) The Author(s). 2018 Open Access This article is distributed under the terms of the Creative Commons Attribution 4.0 International License (http://creativecommons.org/licenses/by/4.0/), which permits unrestricted use, distribution, and reproduction in any medium, provided you give appropriate credit to the original author(s) and the source, provide a link to the Creative Commons license, and indicate if changes were made. The Creative Commons Public Domain Dedication waiver (http://creativecommons.org/publicdomain/zero/1.0/) applies to the data made available in this article, unless otherwise stated. 


\section{Background}

Glomerulonephritis (GN) is a major cause of end stage renal failure (ESRF) [1]. It represents the primary cause of end stage renal disease (ESRF) for $25 \%$ of the dialysis population [1] and $45 \%$ of the transplant population (Table 1). For patients with GN requiring renal replacement therapy, kidney transplantation is associated with superior outcomes compared with dialysis [2]. However, recurrence of GN is reported in 6-20\% [3-8] of renal allograft recipients depending on duration of follow-up [5], local protocol biopsy practice, and type of primary GN [9]. Furthermore, recurrence of GN has a negative impact on long term graft outcomes with recurrence being the third most common cause of allograft loss after chronic rejection and death $[3,4]$.

The risk of recurrence and its impact on outcomes are important questions for patients and clinicians in considering transplantation. It is unclear how GN recurrence in a first allograft impacts the risks of recurrence and graft survival in subsequent kidney transplants. To determine the consequences in subsequent kidney allografts we examined the incidence and timing of allograft loss due to biopsy-proven recurrence of GN from the Australia and New Zealand Dialysis and Transplant registry (ANZDATA), evaluating risk factors for allograft loss and the impact of GN recurrence in first kidney allografts on subsequent kidney transplants based on 28 years of transplant registry data.

\section{Methods}

\section{Study population}

Data were extracted from ANZDATA for all renal allografts transplanted for patients with a biopsy proven GN between 1985 and 2013 within Australia and New Zealand. Transplants included first, second, or subsequent transplants. In analyses of "Primary Glomerulonephritis" we included only patients with IgA Nephropathy (IGAN), Focal Segmental Glomerulosclerosis (FSGS), Membranous Nephropathy (MN) and Mesangiocapillary Glomerulonephritis (MCGN), excluding "Other GN". All data is reported annually by individual units and the diagnosis of GN is determined by individual units.

\section{Outcomes}

Primary end points were recurrence of GN and death censored allograft loss until December 2013. Recurrence of $\mathrm{GN}$ is reported by individual units with the date of the biopsy. Death censored allograft loss is defined by the need to commence renal replacement therapy by dialysis or repeat kidney transplantation.

\section{Statistics}

All statistical analyses were carried out in $\mathrm{R}$ [10]. Baseline characteristics among groups were assessed using Pearson's chi-square test and one way ANOVA. Significance of non-parametric data was assessed by Mann-Whitney $U$ and categorical data by $\chi^{2}$. Survival analyses were performed in $\mathrm{R}$, using the Surv function from the survival library [11] and the npsurv and survplot functions from the rms library [12]. Graphs are plotted with $95 \%$ confidence intervals. Cox proportional hazards ratios are calculated using the coxph function from the survival library, with testing of assumptions in cox.zph. The Cox model included donor category, age category, dialysis vintage, peak PRA, gender, recurrence, and total ischemic time. A time interaction variable was introduced for GN recurrence in the cox model for death censored graft survival.

\section{Results}

A total of 16,023 kidney transplants were performed during the period 1985-2013 of which 7236 (45.16\%) were attributed to biopsy-proven GN. As expected, the majority of transplants performed for GN within this period were primary allografts $(n=6475,89.5 \%)$ followed by secondary $(n=686,9.5 \%)$ and subsequent allografts $(n=75,1 \%)$. Median follow up was 6.1 years (IQR 2.46-11.47). Baseline demographics are summarised in Table 1.

Incidence and time to post-transplant glomerulonephritis recurrence

GN recurrence occurred in 511 (7.06\%) of any kidney allografts in patients with GN within the follow-up period. In GN attributed to a primary GN (IGAN, FSGS, MN or MCGN) there were 424 recurrences from a total of 4025 transplants (10.5\%). Overall, recurrence in either primary or secondary allograft was highest for MCGN (15.52\%) followed by $\mathrm{MN}(12.30 \%)$ and lowest in Other GN (2.71\%) (Table 2).

Primary allograft recurrence was highest for MCGN $(n=48,16.6 \%)$. In comparison to previous reports, FSGS was reported with the lowest recurrence rates $(n=101,10.4 \%)$. For second allografts recurrence was greatest for FSGS (19.3\%) followed by MCGN (10.9\%) (Table 2).

To determine whether time to recurrence differed by GN, we examined recurrence free survival in any allograft for each GN (Fig. 1.) When compared with IGAN, recurrence of FSGS and MCGN occurred with significantly shorter median times to recurrence (0.56 vs 4.63 years, FSGS vs IGAN, $p<0.0001,1.87$ vs 4.63 years, MCGN vs IGAN, $p=0.0003$ ) (Table 3 ). Recurrence rates at 5 and 10 years are shown in Table 4 . 


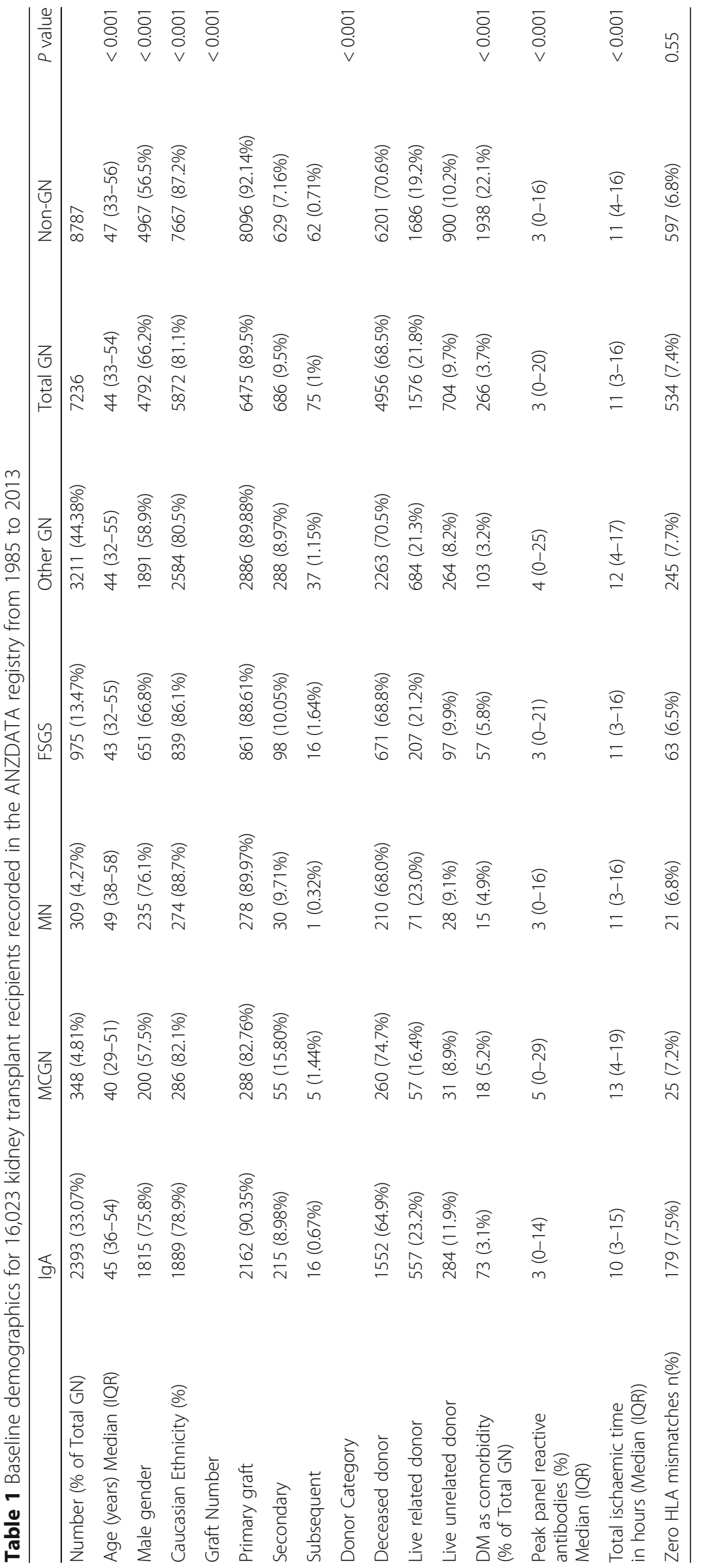




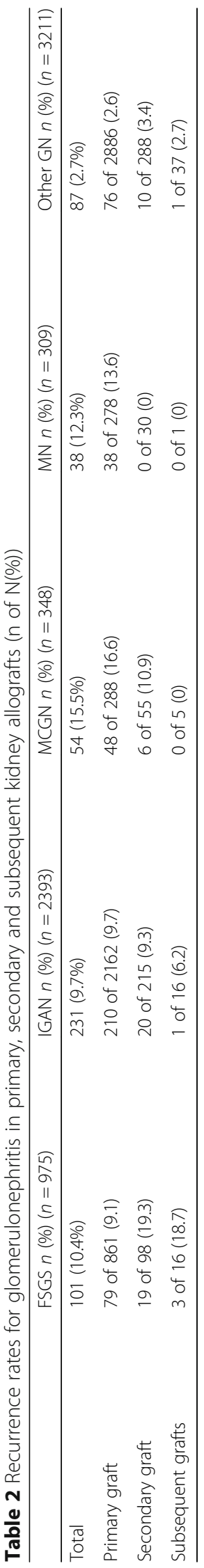




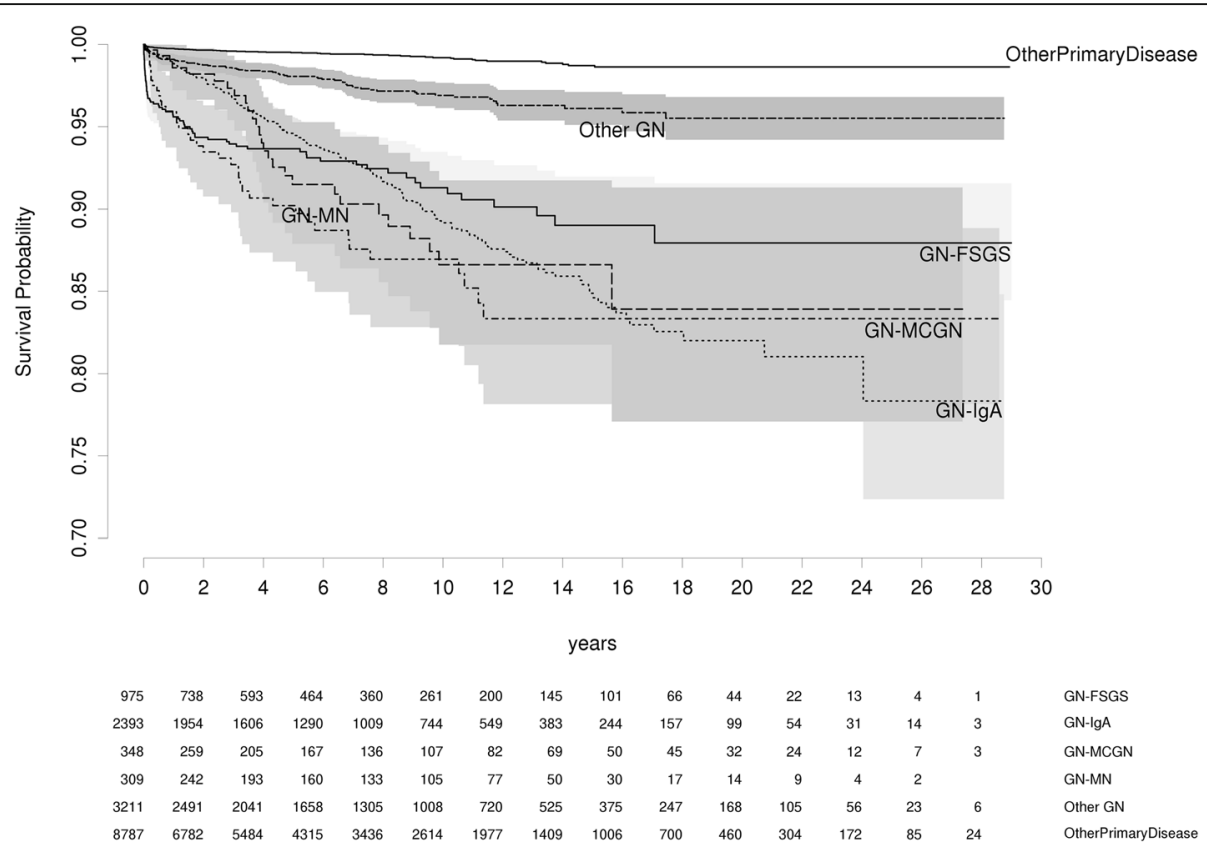

Fig. 1 Recurrence free survival for each disease category

\section{Recurrence of FSGS in a primary graft increases risk of recurrence in a subsequent graft}

We then examined the implication of GN recurrence in a first allograft for risk of recurrence in a subsequent transplant. We compared rates of recurrence in secondary grafts between patients with or without recurrence in their primary kidney allograft. FSGS has a significantly higher risk of recurrence in secondary grafts after a prior recurrence (RR 5.70, 95 CI: 2.41-13.5, $p<0.001$ ) (Table 5). Recurrence in a primary allograft was not associated with increased risk of recurrence in subsequent kidney allografts in IGAN (RR: 1.24, 95 CI: 0.53-2.91, $p=0.62$ ) or MCGN (RR: 1.79, 95 CI: 0.37-8.63 $p=0.5$ ) (Table 5). Therefore, recurrence in primary kidney allografts is associated with increased risks of recurrence in secondary allografts for patients with FSGS, but not IGAN nor MCGN.

Table 3 Number of patients with documented time to recurrence. Time to recurrence in Years

\begin{tabular}{lll}
\hline & Number with time to recurrence & Median (IQR) Years \\
\hline FSGS & 72 of 102 & $0.56(0.05-2.91)$ \\
IGAN & 201 of 231 & $4.63(2.12-8.66)$ \\
MCGN & 38 of 54 & $1.87(0.52-4.88)$ \\
MN & 27 of 38 & $3.93(2.91-6.47)$ \\
Other & 76 of 87 & $2.52(0.39-6.66)$ \\
\hline
\end{tabular}

\section{GN recurrence reduces kidney allograft survival}

To estimate the effect of recurrence on allograft survival, we examined survival in kidney allografts with or without recurrence. As expected, death censored graft survival is significantly reduced in patients with recurrence of any GN (HR 3.1, 95CI: 2.38-3.97 $p<0.001$ ) (Fig. 2) and for each primary GN (Fig. 3, Table 6). In all GN except FSGS, graft survival is superior for recurrent patients over the first twelve months. This most likely reflects ascertainment bias through selection against patients with early graft failure from early acute rejection or surgical complications. The earlier recurrence of FSGS in the post-operative period competes with the risks of these early causes of graft failure. Death censored graft survival at 5 and 10 years for recurrent and non-recurrent patients are presented in Table 6 with hazard ratios for each GN in Table 7.

Table 4 Recurrence rates for Primary GN at 5 and 10 years posttransplant

\begin{tabular}{lll}
\hline & 5 years & 10 years \\
\hline FSGS & $6.3 \%(4.7-7.9)$ & $8.7 \%(6.5-10.8)$ \\
IGAN & $5.4 \%(4.3-6.5)$ & $10.8 \%(9.2-12.5)$ \\
MCGN & $9.8 \%(6.2-13.2)$ & $13.1 \%(8.7-17.2)$ \\
MN & $8.5 \%(4.7-12.1)$ & $13.4 \%(8.3-18.2)$ \\
Other & $1.9 \%(1.4-2.5 \%)$ & $3.1 \%(2.3-3.9)$ \\
\hline
\end{tabular}


Table $\mathbf{5}$ Incidence of GN recurrence in secondary grafts analysed by outcome of primary grafts

\begin{tabular}{|c|c|c|c|}
\hline \multicolumn{4}{|c|}{ ncidence of GN recurrence in secondary grafts } \\
\hline & Recurrence & Non-Recurrence & $\begin{array}{l}\text { Risk Ratio of recurrence in secondary } \\
\text { graft after recurrence in the first }\end{array}$ \\
\hline FSGS & 13 of $27(48.2 \%)$ & 6 of $71(8.5 \%)$ & $5.70(2.41-13.5)$ \\
\hline IGAN & 8 of $75(10.6 \%)$ & 12 of $140(8.6 \%)$ & $1.24(0.53-2.91)$ \\
\hline MCGN & 2 of $12(16.6 \%)$ & 4 of $43(9.3 \%)$ & $1.79(0.37-8.63)$ \\
\hline MN & 0 of $8(0 \%)$ & 0 of $22(0 \%)$ & NA \\
\hline
\end{tabular}

Whilst death censored graft survival is reduced in GN recurrence, the overall effect of recurrence is more complex. We performed a competing risk analysis of death and graft failure by GN recurrence, which showed the recurrent patients have a lower risk of death but a higher risk of graft failure (Fig. 4). However, adjusting for age, gender and the lower prevalence of diabetes accounted for the lower risk of death in patients with GN recurrence (data not shown).

\section{Transplant outcomes and graft loss}

One thousand nine hundred fourteen primary GN patients lost their graft of whom 329 (17.19\%) had a documented recurrence of GN. Causes of graft loss have been analysed in GN and non-GN patients (Table 8 ) and in patients with and without GN recurrence (Table 9). GN patients have similar causes of graft loss to non-GN patients excluding loss due to GN recurrence. This is balanced by a reduction in loss due to rejection and chronic allograft nephropathy. Patients with recurrence have a significantly higher risk of graft failure due to GN (57\%) and a lower risk of loss due to rejection when compared with GN patients without recurrence.

\section{Age, gender, donor status and dialysis vintage are predictors of $\mathrm{GN}$ recurrence}

We then examined variables influencing recurrence of GN. By univariate analysis, male gender, age below 50 years, duration on dialysis less than 5 years before transplant and peak panel reactive antibodies (PRA) were significantly associated with GN recurrence (Fig. 5). Multivariate analysis confirmed higher risk of recurrence evident for patients under 50 years of age (HR $1.5995 \mathrm{CI}$ : 1.21-2.09 $p<0.001$ ), related kidney donation (HR 1.68, 95 CI: $1.31-$ $2.15, p<0.001$ ) and male gender (HR 1.60, 95 CI: $1.23-$ 2.08, $p<0.001$ ) but dialysis vintage was not associated. Therefore, age below 50 years, male gender and a

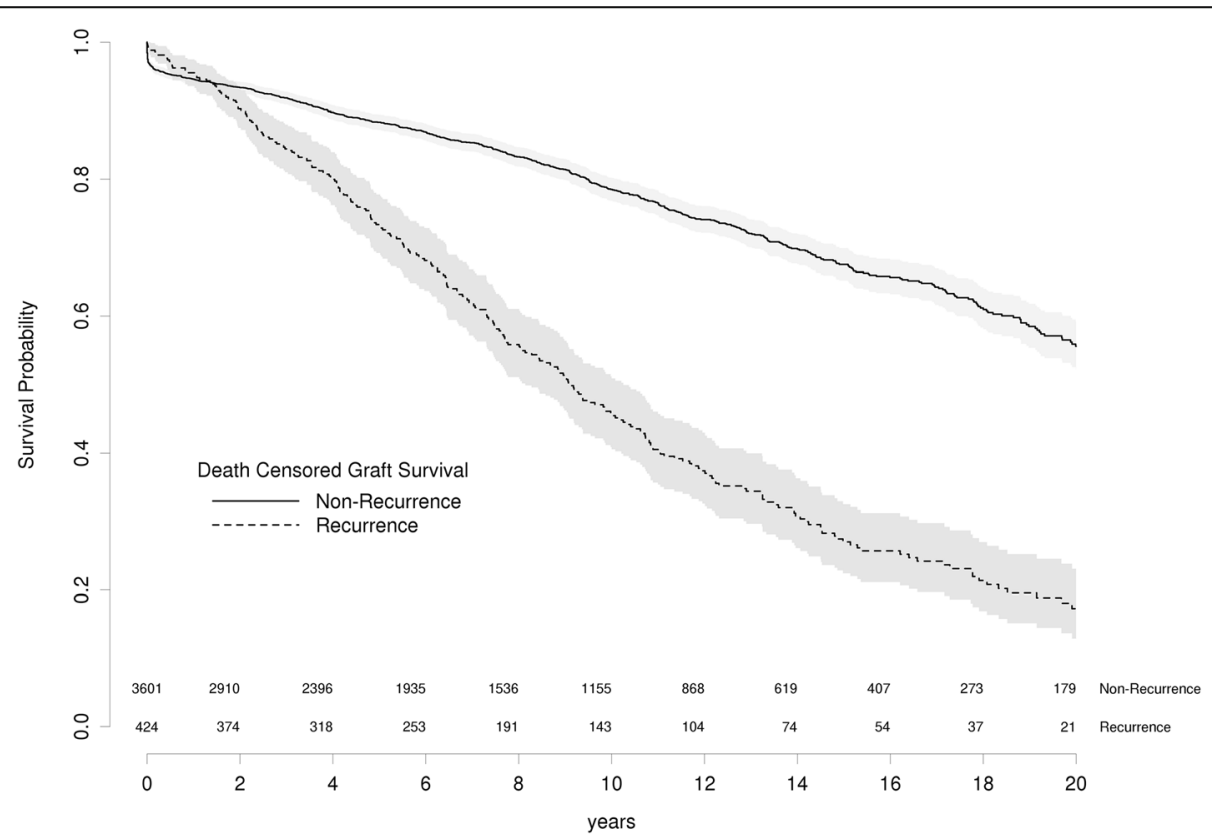

Fig. 2 Death Censored Graft Survival in primary GN patients 


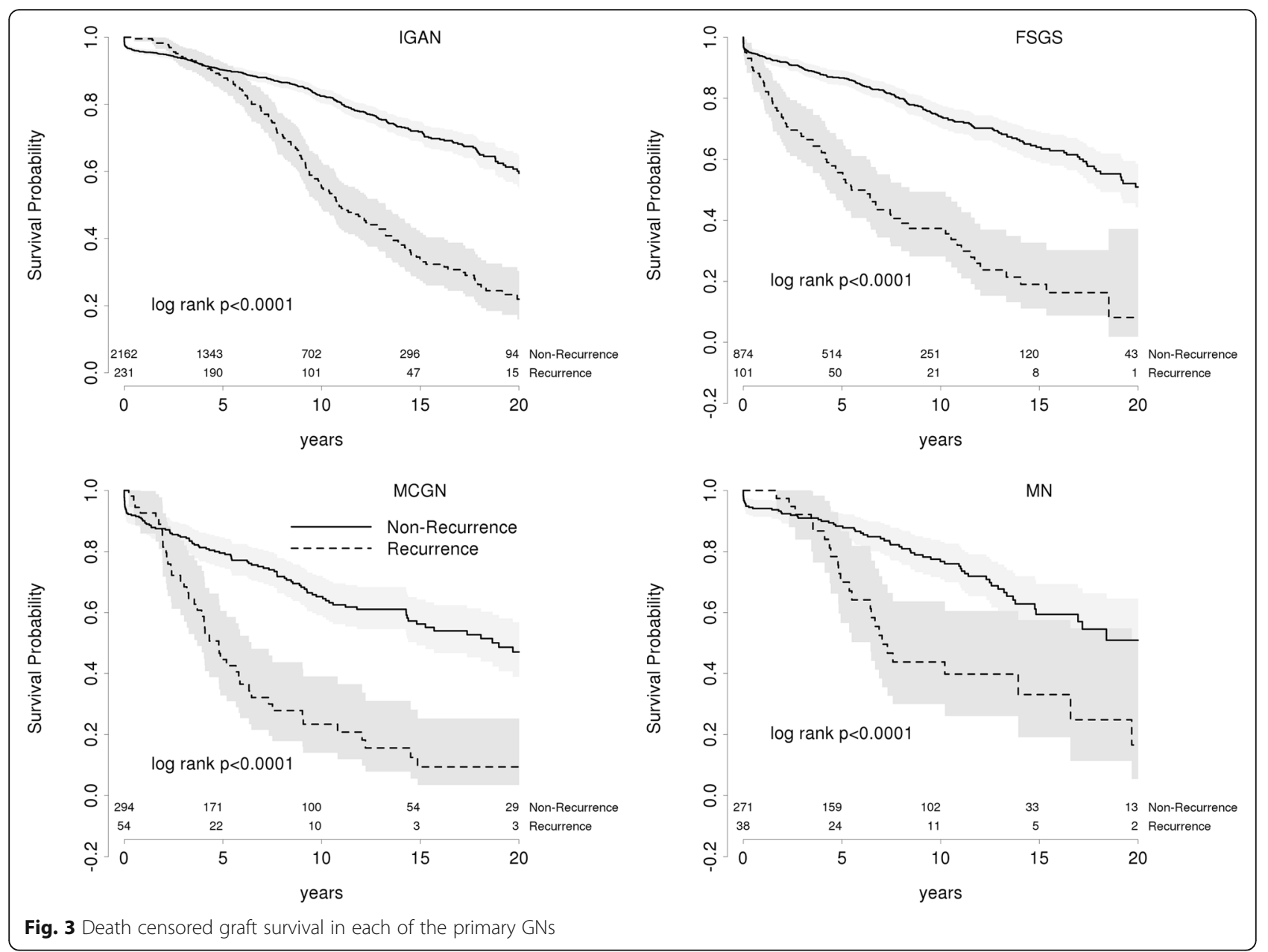

related donor are associated with increased risks of recurrence.

\section{Discussion}

Our study reaffirms unique patterns of recurrence and disease progression for each of the four primary GNs. In general, recurrence rates are low occurring in 10-16\% of patients over 12 years. Of the four primary GNs, FSGS has the more aggressive natural history. Patients with FSGS had significantly faster onset of recurrence than the other primary GNs. This study reinforces the risk of early and aggressive recurrence associated with FSGS and MCGN which has been reported elsewhere [4, 13-15].

FSGS was the only GN in which recurrence in a first transplant predicts greater risk in subsequent grafts, although the rate of recurrence in the primary allograft was lower than expected. This may reflect ascertainment or classification bias in the diagnosis of rapidly failing primary allografts. In IGAN and MCGN, recurrence in the primary allograft does not appear to predict the risk of disease recurrence in subsequent allografts. Previous reports by Ohmacht et al. suggested higher risks in IGAN for second grafts [16]. Freese reported 6 patients with IGAN in a

Table 6 Death Censored Graft Survival at 5 and 10 years post transplant in patients with and without GN recurrence

\begin{tabular}{lllll}
\hline & 5 years & & 10 years & \\
\hline & Recurrent & Non-recurrent & Recurrent & Non-recurrent \\
IGAN & $88.3(84.1-92.6)$ & $90.2(88.9-91.6)$ & $55.1(48.5-62.5)$ & $82.4(80.4-84.5)$ \\
FSGS & $55.6(46.5-66.5)$ & $86.6(84.3-89.1)$ & $37.3(28.2-49.4)$ & $74.4(70.5-77.9)$ \\
MCGN & $44.6(32.8-60.5)$ & $79.5(74.8-84.6)$ & $23.4(14-39)$ & $65.2(59.1-0.72)$ \\
MN & $70(56.6-86.6)$ & $88.3(84.2-92.5)$ & $43.7(30-63.7)$ & $76.8(70.8-83.2)$ \\
\hline
\end{tabular}


Table 7 Hazard ratios for death censored graft survival in patients with recurrent GN

\begin{tabular}{lll}
\hline GN & HR $(95 \% \mathrm{Cl})$ & $P$ value \\
\hline IgA & $4.49(2.70-7.47)$ & $<0.001$ \\
FSGS & $1.83(1.12-3.0)$ & 0.02 \\
MCGN & $3.14(2.16-4.57)$ & $<0.001$ \\
MN & $2.20(1.26-3.86)$ & 0.03 \\
\hline
\end{tabular}

transplant undergoing a second graft [17], wherein one patient developed recurrence. The largest series of recurrence for any GN reported so far came from Briggs with 23 of 48 patients (48\%) developing graft failure due to recurrence in second grafts [13]. These were in all forms of GN including childhood FSGS and MCGN, which makes it difficult to interpret.

In examining variables that may contribute to recurrence, our study indicates that age and male gender are associated with greater risks of GN recurrence. Interestingly, living related donation was also significantly associated with increased risks of GN recurrence suggesting a genetic contribution to GN recurrence.

Other reports present conflicting observations of the effect of recurrence on allograft survival. Moroni et al. [18], Ponticelli [19] and Kim [20] reported high rates of $\mathrm{GN}$ recurrence but no effect of recurrence on allograft survival. These studies all suffered from relatively low study numbers. Moriyama studied 49 patients with IGAN who were transplanted. All patients had their allografts biopsied on implantation, 13 of them were shown to have latent IgA deposits already [21]. They reported a $26 \%$ IGAN recurrence rate at 5 years, higher than ours, but interestingly demonstrated that latent IgA deposition in the graft at donation was a significant risk for both $\mathrm{GN}$ recurrence and subsequent graft failure Conversely, Bumgardner reported recurrence of IGAN in 18 of 61 transplants
Table 8 Causes of graft failure in patients with and without GN

\begin{tabular}{lll}
\hline Cause & GN Patients & Other Patients \\
\hline ATN & $0.50 \%$ & $1.00 \%$ \\
BK & $1.00 \%$ & $0.80 \%$ \\
CAN & $55.40 \%$ & $59.50 \%$ \\
Cortical Necrosis & $1.30 \%$ & $2.10 \%$ \\
Donor Malignancy & $0.50 \%$ & $0.30 \%$ \\
Drugs & $1.30 \%$ & $1.20 \%$ \\
Embolus & $0.30 \%$ & $0.30 \%$ \\
GN & $13.50 \%$ & $1.80 \%$ \\
Haemorrhage & $0.90 \%$ & $1.20 \%$ \\
HUS & $0.70 \%$ & $1.20 \%$ \\
Infection & $1.10 \%$ & $1.30 \%$ \\
Malignancy & $0.40 \%$ & $0.60 \%$ \\
Non-compliance & $3.30 \%$ & $3.70 \%$ \\
Other & $2.50 \%$ & $3.30 \%$ \\
Rejection & $10.90 \%$ & $13.80 \%$ \\
Urological & $0.30 \%$ & $0.70 \%$ \\
Vascular & $6.10 \%$ & $7.10 \%$ \\
\hline
\end{tabular}

with a mean follow-up time of 5 years with significant graft loss due to recurrence [22].

In this study, recurrence of GN was associated with a significant reduction in kidney allograft survival. Our survival analyses suggest improved graft survival in the first year of transplant for each primary GN except FSGS. This likely reflects ascertainment bias as patients with early graft failure from early acute rejection or surgical complications will therefore not be diagnosed with earlier recurrence of FSGS in the post-operative period.

Previous registry studies have suggested that graft half-life is less than 5 years with GN recurrence with a relative risk of 1.9 calculated for graft loss [23]. In this study, grafts with GN recurrence have a half life

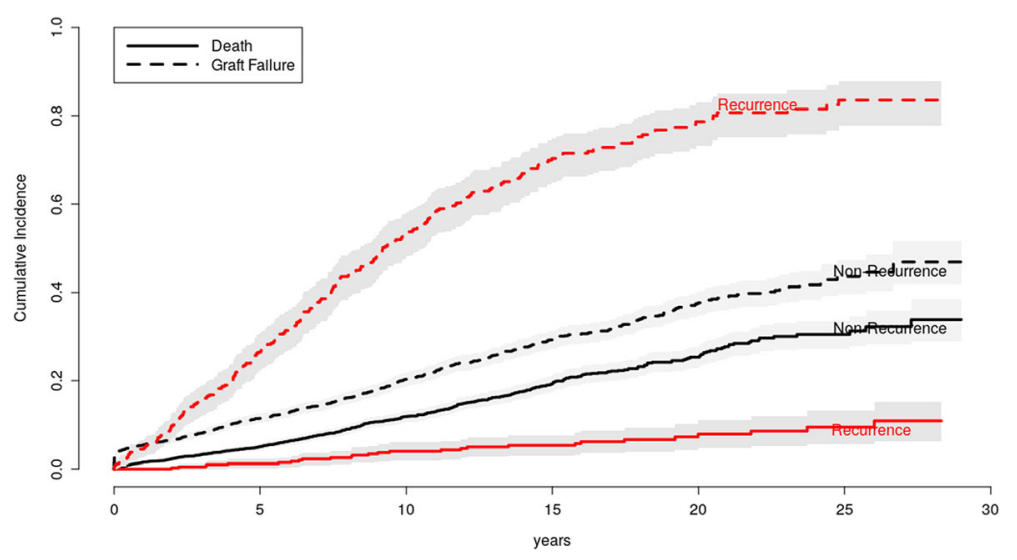

Fig. 4 Competing risk analysis of death and graft failure. Patients with recurrent GN have a lower risk of death but a higher risk of graft failure 
Table 9 Causes of graft failure in GN patients with and without recurrence

\begin{tabular}{lll}
\hline Cause & Non-Recurrence & Recurrence \\
\hline ATN & $0.80 \%$ & $0.00 \%$ \\
BK & $1.00 \%$ & $0.30 \%$ \\
CAN & $60.50 \%$ & $30.30 \%$ \\
Cortical Necrosis & $1.10 \%$ & $0.30 \%$ \\
Donor Malignancy & $0.40 \%$ & $0.00 \%$ \\
Drugs & $1.30 \%$ & $0.80 \%$ \\
Embolus & $0.30 \%$ & $0.30 \%$ \\
GN & $2.30 \%$ & $55.00 \%$ \\
Haemorrhage & $1.20 \%$ & $0.00 \%$ \\
HUS & $0.70 \%$ & $3.30 \%$ \\
Infection & $1.30 \%$ & $0.00 \%$ \\
Malignancy & $0.50 \%$ & $0.50 \%$ \\
Non-compliance & $3.80 \%$ & $0.80 \%$ \\
Other & $2.70 \%$ & $5.40 \%$ \\
Rejection & $14.20 \%$ & $2.60 \%$ \\
Urological & $0.60 \%$ & $0.00 \%$ \\
Vascular & $7.30 \%$ & $0.50 \%$ \\
\hline
\end{tabular}

of approximately 10 years and a Hazard Ratio of 3.1 for death censored graft failure compared to non-recurrent grafts. MCGN, MN and FSGS tend to have shorter graft half lives. We are in agreement with a more recent single centre study though their analysis did not show GN recurrence as a risk for death censored graft failure [18]. Moroni analysed 190 IGAN transplants and compared them with 380 non-diabetic controls, suggesting that IGAN patients had worse survival than the controls but recurrence of disease had little impact on graft survival for the first 10 years [24]. In chinese patients with IGAN, Choy reported a $20 \%$ recurrence rate at 10 years but with little impact on graft survival [25]. The estimates in our study are based on a much larger cohort and are likely to be more robust.

The strength of our analysis lies in the large database with a well-characterised study population and complete dataset with up to 28 years follow-up. There are limitations inherent in Registry analysis in terms of heterogeneity of clinical practice across contributing units. Over

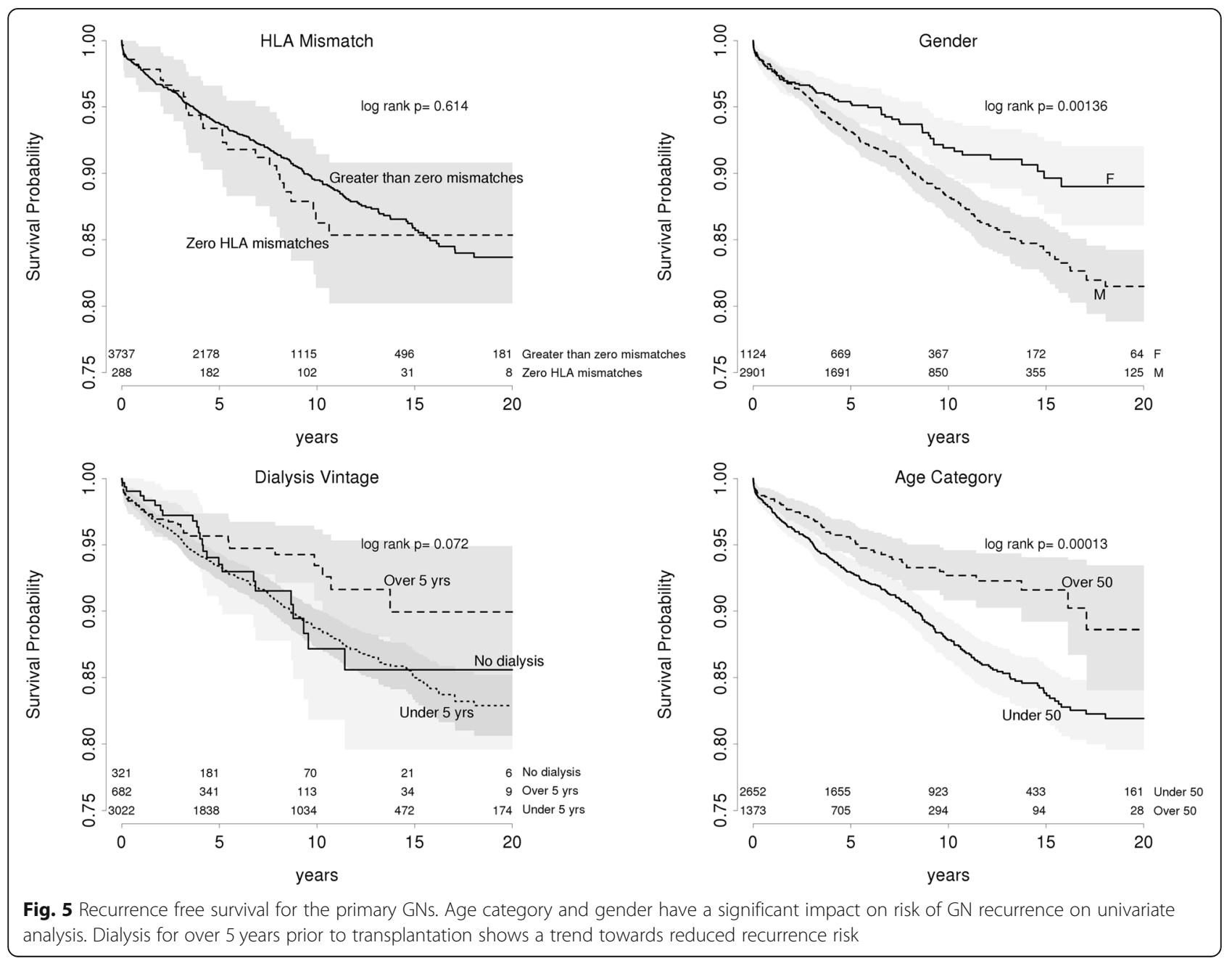


the period of data collection, disease definition has changed. FSGS and MCGN are now regarded as multiple disease entities. It is not possible to retrospectively reclassify these diseases and it is important to keep this in mind in interpreting our data. Also the diagnosis of recurrent GN was conducted by each hospital. They was no independent examination of the diagnosis possible. Also the reason for the kidney biopsy was not included in this analysis and indeed was not available for this dataset. Despite these limitations, this study represents the most complete and well-populated registry analysis to date.

The increasing use of protocol biopsies is likely to increase reported risk of GN recurrence but should be viewed by clinicians with caution. This aggressive approach to transplant followup has yet to be shown to alter hard outcomes and, in the setting of recurrent GN, may be potentially misleading. The interpretation of immunoglobulin deposits in a donor kidney in the absence of clinical disease is far from clear. As demonstrated in the implantation biopsies of donor kidneys [21], the presence of IgA deposits in a kidney does not necessarily imply disease recurrence in the recipient and may instead be a marker of the donor's genetic predisposition to renal disease. At a minimum, the research community needs to establish that protocol biopsy data is clinically meaningful in terms of recurrent glomerulonephritis.

\section{Conclusions}

Primary GN recurs in 10-16\% of renal allografts over a 15 year period. FSGS and MCGN tend to recur earlier than IGAN. After recurrence, graft survival is significantly reduced. Transplants performed after a previous recurrence have similar risks of recurrence, except in the case of FSGS, which is more likely to recur. Patients and clinicians need to be aware of the risks of recurrence and its consequences.

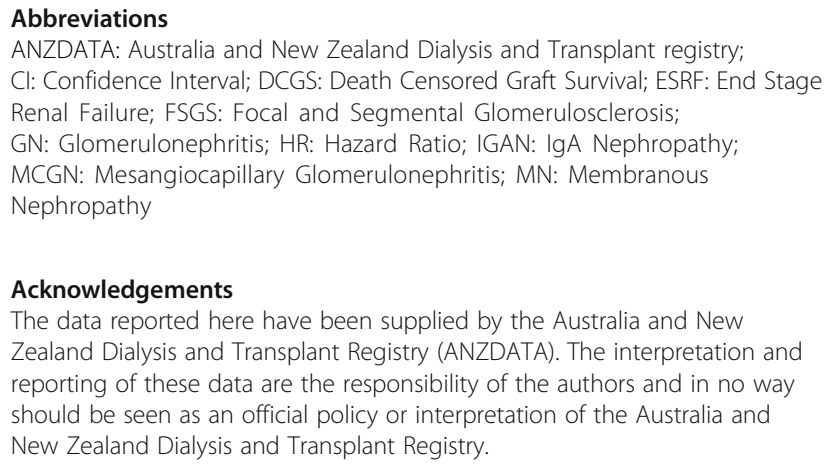

\section{Acknowledgements}

The data reported here have been supplied by the Australia and New Zealand Dialysis and Transplant Registry (ANZDATA). The interpretation and reporting of these data are the responsibility of the authors and in no way should be seen as an official policy or interpretation of the Australia and New Zealand Dialysis and Transplant Registry.

\section{Ethics and consent to participate}

No specific ethics approval has been sought for this study. Data was sought under standard processes for the ANZDATA registry. All patients consent to have their data collected and analysed prior to data entry in the Registry.
Funding

Not applicable.

\begin{abstract}
Availability of data and materials
The datasets generated for this study are available in the LabArchives repository at doi: https://doi.org/10.6070/H4Z60M30
\end{abstract}

\section{Authors' contributions}

AK contributed data acquisition, data analysis and interpretation, drafting and revision of the manuscript. SJ contributed study conception, data analysis and interpretation, drafting and revision of the manuscript. GW contributed study conception, data acquisition, data analysis and interpretation, drafting and revision of the manuscript. All authors have approved the final manuscript and take full responsibility for its content and accuracy.

\section{Consent for publication}

The manuscript contains no indentifiable or re-identifiable data, therefore no consent to publish was sought.

\section{Competing interests}

All authors declare that they have no competing interest.

\section{Publisher's Note}

Springer Nature remains neutral with regard to jurisdictional claims in published maps and institutional affiliations.

\section{Author details}

${ }^{1}$ Department of Renal Medicine, The Canberra Hospital, PO Box 11, Woden, Canberra ACT 2605, Australia. ²Department of Immunology and Infectious Diseases, John Curtin School of Medical Research, Australian National University, Canberra, Australia. ${ }^{3}$ Australian National University Medical School, Canberra, Australia. ${ }^{4}$ ANZDATA Registry, Adelaide 5000, Australia.

Received: 13 July 2018 Accepted: 12 November 2018 Published online: 03 December 2018

References

1. Schena FP. Epidemiology of end-stage renal disease: international comparisons of renal replacement therapy. Kidney Int. 2000;57:S39-45.

2. McDonald SP, Russ GR. Survival of recipients of cadaveric kidney transplants compared with those receiving dialysis treatment in Australia and New Zealand, 1991-2001. Nephrol Dial Transplant. 2002;17:2212-9.

3. Floege J. Recurrent glomerulonephritis following renal transplantation: an update. Nephrol Dial Transplant. 2003;18:1260-5.

4. Briganti EM, Russ GR, MCNeil JJ, Atkins RC, Chadban SJ. Risk of renal allograft loss from recurrent glomerulonephritis. N Engl J Med. 2002;347:103-9.

5. Hariharan S, Peddi VR, Savin V, Johnson CP, First MR, Roza AM, et al. Recurrent and de novo renal diseases after renal transplantation: a report from the renal allograft disease registry. Am J Kidney Dis. 1998;31:928-31.

6. Yakupoglu U, Baranowska-Daca E, Rosen D, Barrios R, Suki WN, Truong LD. Post-transplant nephrotic syndrome: a comprehensive clinicopathologic study. Kidney Int. 2004;65:2360-70.

7. Freese PM, Svalander CT, Mölne J, Nyberg G. Renal allograft glomerulopathy and the value of immunohistochemistry. Clin Nephrol. 2004;62:279-86.

8. Chadban SJ. Glomerulonephritis recurrence in the renal graft. J Am Soc Nephrol. 2001;12:394-402

9. Morozumi K, Takeda A, Otsuka Y, Horike K, Gotoh N, Watarai Y. Recurrent glomerular disease after kidney transplantation: an update of selected areas and the impact of protocol biopsy. Nephrology. 2014;19:6-10.

10. R: The R Project for Statistical Computing. [cited 2016 Apr 7]; Available from: https://www.r-project.org/

11. Therneau TM, Thomas Lumley (original S.-> R port and maintainer until 2009). survival: Survival Analysis. 2015 [cited 2016 Apr 7]; Available from: https://cran.r-project.org/web/packages/survival/index.html

12. Jr FEH. rms: Regression Modeling Strategies. 2016 [cited 2016 Apr 7]; Available from: https://cran.r-project.org/web/packages/rms/index.html

13. Briggs JD, Jones E. Recurrence of glomerulonephritis following renal transplantation. Scientific advisory board of the ERA-EDTA registry. European renal association-European Dialysis and transplant association. Nephrology Dialysis. Transplantation. 1999;14:564-5. 
14. Lorenz EC, Sethi S, Leung N, Dispenzieri A, Fervenza FC, Cosio FG. Recurrent membranoproliferative glomerulonephritis after kidney transplantation. Kidney Int. 2010;77:721-8.

15. Green H, Rahamimov R, Rozen-Zvi B, Pertzov B, Tobar A, Lichtenberg S, et al. Recurrent Membranoproliferative glomerulonephritis type I after kidney transplantation: a 17-year single-center experience. Transplantation. 2015;99:1172-7.

16. Ohmacht C, Kliem V, Burg M, Nashan B, Schlitt H-J, Brunkhorst R, et al. Recurrent immunoglobulin a nephropathy after renal transplantation: a significant contributor to graft Loss1. Transplantation. 1997;64:1493-6.

17. Freese P, Svalander C, Nordén G, Nyberg G. Clinical risk factors for recurrence of IgA nephropathy. Clin Transpl. 1999;13:313-7.

18. Moroni G, Longhi S, Quaglini S, Rognoni C, Simonini P, Binda V, et al. The impact of recurrence of primary glomerulonephritis on renal allograft outcome. Clin Transpl. 2014;28:368-76.

19. Ponticelli C, Glassock RJ. Posttransplant recurrence of primary glomerulonephritis. Clin J Am Soc Nephrol. 2010;5:2363-72.

20. Kim YS, Moon Jl, Jeong HJ, Kim MS, Kim SI, Choi KH, et al. Live donor renal allograft in end-stage renal failure patients from immunoglobulin a Nephropathy1, 2. Transplantation. 2001;71:233-8.

21. Moriyama T, Nitta K, Suzuki K, Honda K, Horita S, Uchida K, et al. Latent IgA deposition from donor kidney is the major risk factor for recurrent IgA nephropathy in renal transplantation. Clin Transpl. 2005;19:41-8.

22. Bumgardner GL, Amend WC, Ascher NL, Vincenti FG. Single-center longterm results of renal transplantation for IgA nephropathy. Transplantation. 1998:65:1053-60

23. Hariharan S, Adams MB, Brennan DC, Davis CL, First MR, Johnson CP, et al. recurrent and de novo glomerular disease after renal transplantation: a report from renal allograft disease registry (RADR) 1, 2. Transplantation. 1999;68:635-41.

24. Moroni G, Gallelli B, Quaglini S, Leoni A, Banfi G, Passerini P, et al. Long-term outcome of renal transplantation in patients with idiopathic membranous glomerulonephritis (MN). Nephrol Dial Transplant. 2010;25:3408-15.

25. Choy BY, Chan TM, Lo SK, Lo WK, Lai KN. Renal transplantation in patients with primary immunoglobulin a nephropathy. Nephrol Dial Transplant. 2003;18:2399-404.

Ready to submit your research? Choose BMC and benefit from:

- fast, convenient online submission

- thorough peer review by experienced researchers in your field

- rapid publication on acceptance

- support for research data, including large and complex data types

- gold Open Access which fosters wider collaboration and increased citations

- maximum visibility for your research: over $100 \mathrm{M}$ website views per year

At $\mathrm{BMC}$, research is always in progress.

Learn more biomedcentral.com/submissions 